\title{
Therapeutic strategies for the clinical blockade of IL-6/gp130 signaling
}

\author{
Simon A. Jones, ${ }^{1}$ Jürgen Scheller, ${ }^{2}$ and Stefan Rose-John ${ }^{3}$
}

\begin{abstract}
1Department of Infection, Immunity and Biochemistry, The School of Medicine, Cardiff University, Cardiff, United Kingdom. 2Institute of Biochemistry and Molecular Biology II, Medical Faculty, Heinrich-Heine-University, Düsseldorf, Germany. ${ }^{3}$ Institute of Biochemistry, Christian-Albrechts-University, Kiel, Germany.
\end{abstract}

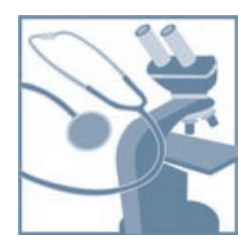

\begin{abstract}
The successful treatment of certain autoimmune conditions with the humanized anti-IL-6 receptor (IL-6R) antibody tocilizumab has emphasized the clinical importance of cytokines that signal through the $\beta$-receptor subunit glycoprotein 130 (gp130). In this Review, we explore how gp130 signaling controls disease progression and examine why IL-6 has a special role among these cytokines as an inflammatory regulator. Attention will be given to the role of the soluble IL-6R, and we will provide a perspective into the clinical blockade of IL-6 activity in autoimmunity, inflammation, and cancer.
\end{abstract}

\section{Introduction}

Inflammation is not a disease but a consequence of disease and is the body's defense against infection or injury. When effective, the inflammatory response ensures successful resolution of the condition and forms part of the normal healing process. Regulation of this response is centrally controlled by cytokine-driven communication, which governs both innate and adaptive immunity. In more progressive chronic inflammatory diseases, the natural course of inflammation is lost, resulting in disease progression instead of protection. The successful treatment of inflammatory conditions with biologics that block cytokine activity indicates that imbalanced proinflammatory and antiinflammatory cytokine responses contribute to the induction of autoimmunity, chronic inflammation, and associated tissue damage $(1,2)$. Although these drugs have provided considerable clinical benefit, we have yet to fully understand how the cytokine network becomes distorted to drive chronic inflammation rather than competent host defense (2).

Preclinical models have emphasized the involvement of numerous cytokines in the pathology of various inflammatory diseases and cancers. As a consequence, cytokines have become major therapeutic targets for clinical intervention. For example, mAbs that target TNF- $\alpha$ are now the standard treatment for patients with chronic inflammatory arthritis, and alternative therapies, which target other cytokines, are also emerging in routine clinical practice $(1,2)$. These agents work by either targeting the cytokine directly or by inhibiting cytokine binding to their specific receptors on the surface of cells. In this regard, they are designed to prevent cytokine signaling within cells. This fundamental mode of action has also fuelled renewed excitement about the possibility of blocking certain intracellular cytokine signaling pathways with small molecule inhibitors. The challenge is to identify which cytokine or signaling molecule represents the most appropriate intervention target for a particular patient group. In this regard, a candidate pharmaceutical needs to block a sufficiently broad number of pathological processes associated with the disease

Conflict of interest: Stefan Rose-John is an inventor on the patent describing the function of sgp $130 \mathrm{Fc}$. He is also a shareholder of the CONARIS Research Institute (Kiel, Germany), which is commercially developing sgp $130 \mathrm{Fc}$ as a therapy for inflammatory diseases. Simon A. Jones has a consultancy agreement with Roche Pharmaceuticals and advises research relating to the clinical introduction of Actemra/RoActemra. Citation for this article: JClin Invest. 2011;121(9):3375-3383. doi:10.1172/JCI57158. but should also confer a minimal impact on safety concerns, such as infection incidence, cardiovascular risk, and malignancy.

\section{Frontline therapies for chronic inflammation}

Biologics, including the anti-TNF- $\alpha$ agents (e.g., the neutralizing anti-TNF- $\alpha$ antibodies infliximab, adalimumab, golimumab, and certolizumab or the soluble TNF-R2 Fc-fusion protein etanercept), are broadly used drugs that reduce inflammation. The clinical success of these agents has led to a significant research interest in the control of TNF- $\alpha$ processing and signaling (1). Less attention has been given to cytokines that signal through the JAK/STAT pathway (3). However, cytokines that signal via this pathway (e.g., IFN- $\gamma$, GM-CSF, IL-6, IL-10, IL-15, IL-23) have become increasingly linked with the pathogenesis of chronic inflammatory diseases and cancer $(2,4)$. Biologics are now emerging that target these cytokines (e.g., IL-6R blockade by tocilizumab), and selective small molecule JAK inhibitors (e.g., tofacitinib, ruxolitinib) also show favorable phase IIa efficacy in patients with rheumatoid arthritis (5-8). With this rise in the number of biological interventions entering the clinical arena, it has become increasingly important to understand how specific cytokine pathways interface with the inflammatory process to affect disease outcome. This represents a major challenge for both basic and clinical researchers alike. Throughout this Review, we will assess the merits of targeting cytokines that signal via the universal signal-transducing $\beta$-receptor subunit for all IL-6 related cytokines, glycoprotein 130 (gp130).

\section{The involvement of gp130-related cytokines in homeostasis and disease}

gp130 (also known as CD130) is expressed in almost all organs, including heart, kidney, spleen, liver, lung, placenta, and brain, and targeted deletion of the gp130 gene in mice results in embryonic lethality at day 12.5 (9). Histological assessments showed that these animals display hypoplastic ventricular myocardium and greatly reduced numbers of hematopoietic progenitors in the liver and T cells in the thymus (9). These data demonstrate that gp130 plays a fundamental role in development, hematopoiesis, cell survival, and growth.

Although initially identified as the $\beta$ subunit of the IL-6R complex, gp130 also transmits signals for IL-11, IL-27, oncostatin-M 


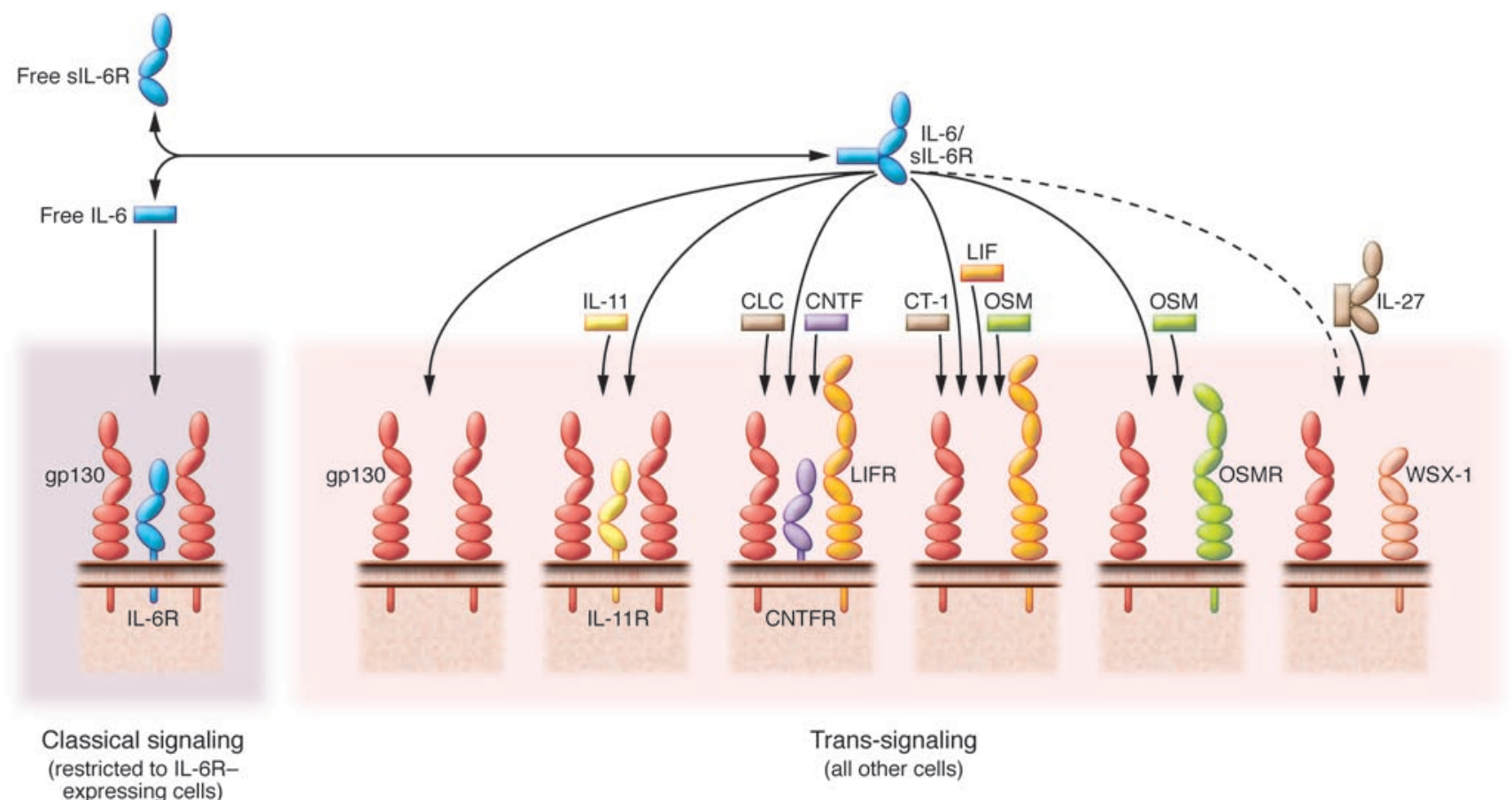

Figure 1

Receptor composition for cytokines signaling via the $\beta$-receptor subunit gp130. gp130-related cytokines (IL-6, IL-11, IL-27, CNTF, CLC, CT-1, LIF, OSM) bind membrane-bound receptor complexes, which all contain the receptor subunit gp130. These cytokines have diverse functions, not limited to but including the induction of acute phase response protein expression; lymphocyte activation; cytoprotective actions on cardiomyocytes, neuronal cells, and hepatocytes; bone metabolism; hematopoiesis; and liver development and regeneration. IL- 6 trans-signaling through the IL-6/sIL-6R complex can fulfill functions of other gp130 cytokines, including blockade of embryonic stem cell differentiation. In addition, since gp130 is expressed in all cells of the body, the IL-6/sIL-6R complex can stimulate cells in additional tissues, which do not express IL-6R, LIF receptor (LIFR), OSMR, IL-11R, CNTF receptor (CNTFR), or the IL-27 receptor subunit WSX-1.

(OSM), ciliary neurotrophic factor (CNTF), cardiotrophin-1 (CT-1), leukemia inhibitory factor (LIF), and the cardiotrophinlike cytokine (CLC) (10-17) (herein referred to as gp130-related cytokines) (Figure 1). Many of these factors elicit similar activities, and the phenotypic characteristics of mice lacking IL-6, IL-11, LIF, or CNTF are less severe than the apparent pleiotropic properties of these mediators would suggest $(13,15)$. In this regard, gp130related cytokines display not only a degree of functional redundancy but also specialization, and some of these functions are not necessary for embryo development.

Studies of mice deficient in gp130-related cytokines demonstrate that these individual family members perform central roles in development and tissue homeostasis. For instance, CNTF, LIF, and CLC act as neurotrophic factors important for the survival and development of motor neurons $(11,12)$. Similarly, OSM, LIF, IL-6, and IL-11 affect multiple hematopoietic processes (including megakaryocyte maturation) and control liver regeneration and hepatocyte proliferation. In some instances these cytokines elicit defined aspects of the same biological process. For example, female IL-11 receptor- $\alpha$-deficient (IL-11R-deficient) mice are infertile due to defective decidualization (18), while expression of LIF in maternal endometrial glands is required for early embryogenesis and embryo implantation (19). These examples help to collectively build the argument that certain gp130-related cytokines govern cellular differentiation and survival in many different organ/tissue/cellular compartments (e.g., LIF, IL-6), while others display more restricted activities affecting a single organ, such as the role of CT- 1 in the heart or CNTF and CLC in neurons.

In addition to the control of homeostatic processes, gp130related cytokines play integral roles in infection, immunity, and inflammation. In particular, IL-6-deficient (IL-6KO) mice are viable but show numerous immunological defects (15). Experimental models show that IL-6KO mice resist the induction of a number of autoimmune conditions (reviewed in ref. 20), although this is not true for animals with defective production of TNF- $\alpha$, a cytokine that frequently cooperates with IL-6 (21-23). For example, IL-6KO mice show limited susceptibility to antigen-induced arthritis (24), collagen-induced arthritis $(25,26)$, experimental autoimmune encephalomyelitis (27), multicentric Castleman disease (28), and pristine-induced plasmacytomas (29). These early studies fuelled interest in IL- 6 as a therapeutic target for autoimmunity and led to the development of agents such as tocilizumab.

\section{Mechanisms of IL-6R signaling}

IL-6 is the archetypal member of the gp130-related cytokine family. IL- 6 activates cells by first binding a nonsignaling $\alpha$-receptor (IL-6R, also known as CD126), which, after dimerization with gp130, leads to activation of receptor-associated kinases (JAK1, JAK2, and Tyk2) within the cell. In turn these lead to phosphorylation of proximal tyrosine residues within the intracellular portion of gp130, and the subsequent control of STAT1 and STAT3 activity, and the Src homology region 2 domain-containing phosphatase 2 cascade (14). 
Importantly, the IL-6R functions as both a membrane-bound protein, which is expressed by hepatocytes and certain inflammatory cells, and a soluble form (sIL-6R), which is readily detected in the circulation and at sites of inflammation (Figure 1). Throughout this Review, we will refer to IL-6 signaling through membranebound IL-6R as classical IL-6R signaling, and IL- 6 trans-signaling will be used to describe activities elicited via sIL-6R (30-32).

Interestingly, although mice deficient in IL-6R (CD126-KO mice) display similar phenotypic characteristics to those of IL-6KO mice $(33,34)$, subtle differences in their functional behavior have been noted. For example, CD126-KO and IL-6KO mice show differences in wound healing (34). Similarly, hepatocyte-specific CD126-KO mice show a lower insulin sensitivity and glucose tolerance, which can be restored by TNF- $\alpha$ neutralization or Kupffer cell depletion (35). The mechanistic basis for these differences currently remains unclear.

In contrast to IL-6KO and CD126-KO strains, mice deficient in related cytokine signaling pathways do not display a similar resistance to autoimmunity. For example in models of arthritis, IL-11R-deficient mice and OSM receptor- $\beta$-deficient (OSMR$\mathrm{KO}$ ) mice develop disease severity comparable to that of wild-type controls (36). Interestingly, IL-27 suppresses development of collagen-induced arthritis and in human cell systems inhibits osteoclastogenesis $(37,38)$. Similarly, IL-11 regulates many antiinflammatory activities in arthritis models $(39,40)$, although trials using recombinant IL-11 in patients with rheumatoid arthritis were suspended due to poor efficacy (41). Other gp130-related cytokines may, however, govern defined aspects of pathology, as is illustrated by the role of IL-11 and OSM in bone turnover (42). Indeed, OSMR $\beta$-KO mice exhibit impaired hepatocyte proliferation (43) and altered monocytic cell trafficking (44), while OSM deficiency is associated with thymic hyperplasia and glomerulonephritis (45). Despite these apparent roles for other gp130-related cytokines in autoimmunity, therapies that target IL-6 or its receptor remain the most developed strategies.

\section{What makes IL-6 an attractive therapeutic target?}

IL-6 is traditionally considered a regulator of acute phase inflammatory responses and a lymphocyte stimulatory factor (14). However, this cytokine also controls homeostatic functions, including regulation of glucose metabolism $(35,46)$ and the hypothalamic-pituitary-adrenal axis (47). IL-6 control of these activities has become an important consideration for IL-6-based interventions, but for the purpose of this Review, we will focus on the inflammatory activities controlled by IL-6. During an inflammatory episode, IL-6 is highly expressed, and circulating levels of this cytokine can rise dramatically from $1-5 \mathrm{pg} / \mathrm{ml}$ to several $\mu \mathrm{g} / \mathrm{ml}$ in certain extreme cases (48). Consequently, IL- 6 is one of the most highly expressed mediators of inflammation. Similar increases during inflammatory episodes are not characteristic of other gp130-related cytokines.

As an inflammatory cytokine, IL- 6 orchestrates chemokinedirected leukocyte trafficking and directs transition from innate to adaptive immunity through regulation of leukocyte activation, differentiation, and proliferation (31). A renewed interest in IL-6 was sparked in 2006, when IL-6, in combination with TGF- $\beta$, was shown to promote the differentiation of IL-17-secreting Thelper (Th17) cells (49). Although Th17 cells are associated with the pathogenesis of various autoimmune/chronic inflammatory states (50), it is presumptuous to assume that anti-IL-6 therapies would be effective because they block Th17 development. Indeed, the anti-IL-6R mAb tocilizumab may be viewed more broadly as a robust inhibitor of IL-6/STAT3 activity. Tocilizumab intervention in rheumatoid arthritis leads to a rapid and sustained improvement in disease activity, a reduction in radiographic joint damage, and inhibition of B cell hyperactivity $(6,51)$. These changes are also associated with a dramatic normalization of the acute phase response (including $\mathrm{C}$-reactive protein [CRP]) and improvements in both pain and fatigue (6). In the following sections, we will highlight some of the complexities associated with IL- 6 biology (for more detailed reviews of IL-6 signaling and its involvement in specific disease, see refs. $4,5,14,31,32,52)$.

\section{Understanding the subtleties of IL-6 biology}

In contrast to the ubiquitous expression of gp130, IL-6R displays a highly restricted expression pattern and is predominantly confined to hepatocytes, leukocyte subsets, and megakaryocytes (5). A similar pattern of expression is also seen with OSMR $\beta$, yet this gp130like protein is also widely expressed in stromal compartments, such as fibroblasts and mesothelial and endothelial cells (44, 53, 54). IL-11R $\alpha$ is largely confined to nonhematopoietic stromal cells, while IL-27R $\alpha$ is restricted to lymphocytes, monocytes, and osteoclasts $(14,55)$. Indeed, IL-27 often opposes the action of IL- 6 and is the only member of the gp130-related cytokine family to predominantly signal via the latent transcription factor STAT1 instead of STAT3 $(14,55)$. In this context, IL-27 acts as a negative regulator of Th17 commitment, while the p28 subunit of IL-27 also antagonizes IL-6-mediated T cell responses (56-58). However, as described earlier, the critical difference between IL- 6 and related cytokines is the existence of a natural sIL-6R, which in humans is generated through differential mRNA splicing but primarily through proteolytic cleavage and subsequent shedding of membrane-bound IL-6R $(30,59)$. The affinity of IL- 6 for sIL-6R is comparable to that of the membrane-bound receptor $(\sim 1 \mathrm{nM})$, and sIL-6R is found at high concentrations in human serum and urine $(60,61)$. The function of sIL-6R is two fold. The formation of an IL-6/sIL-6R complex not only protects IL- 6 and prolongs its circulating half-life (62), but also acts as an agonist capable of directly activating cells through membrane-bound gp130. This trans-signaling enables IL- 6 to activate cells that inherently lack the $\alpha$ subunit for the IL-6R and would normally not respond to this cytokine $(30,63)$. Consequently, IL- 6 trans-signaling may mimic or supplement the paracrine or autocrine activities of certain other gp130-activating cytokines (64). Moreover, since gp130 is ubiquitously expressed, the IL-6/sIL-6R complex can also stimulate cells that are nonresponsive to any other gp130-related cytokine (Figure 1). Although protein-engineering experiments with recombinant soluble receptors for CNTF and IL-11 have recapitulated this signaling mechanism in vitro, IL-6 remains the only example of a cytokine that in vivo uses both classical membrane-bound receptor signaling and trans-signaling through its soluble receptor $(10,63)$. The IL-6/ sIL-6R complex therefore resembles a heterodimeric cytokine akin to either IL-12 (the IL-12p40 subunit shares $60 \%$ identity with sIL-6R) or IL-27 $(14,65)$. Consequently, those who implement therapeutic strategies need to consider the impact of blocking classical membrane-bound signaling and IL-6 trans-signaling (Figure 2). The anti-IL-6R antibody tocilizumab globally blocks IL-6 activities since it inhibits both modes of IL-6 signaling (66).

While research from our groups and others increasingly points toward roles for IL- 6 trans-signaling in regulating processes localized to the site of disease, infection, or injury, less is known about the IL- 6 control of homeostatic processes, such as fatigue, mood, 


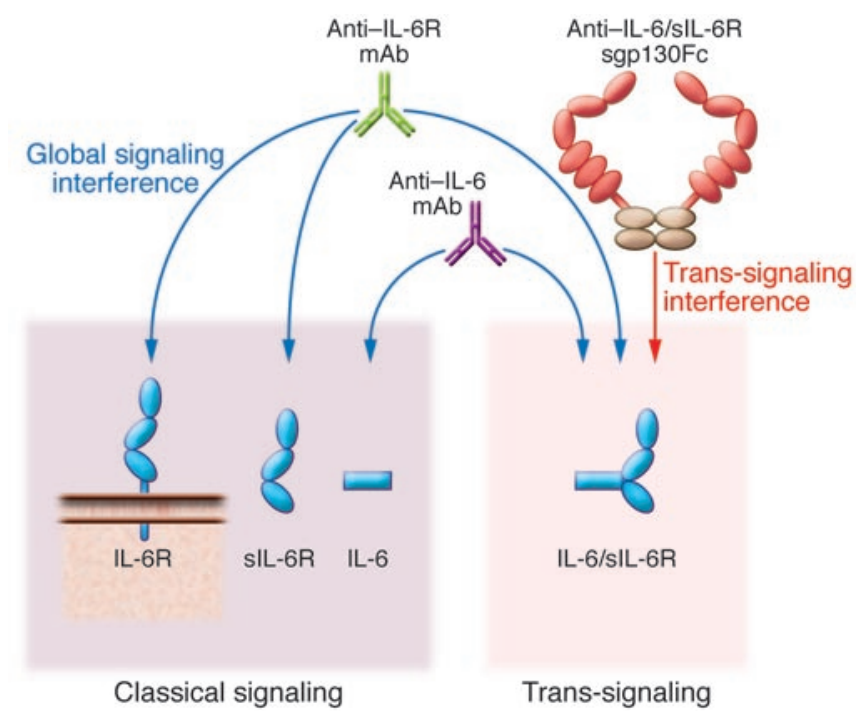

Figure 2

Modes of therapeutic IL-6 blockade. IL-6-neutralizing antibodies (violet; Table 1) block both classical and trans-signaling of IL-6 by interfering with binding of IL- 6 to IL-6R. This might lead to high-level accumulation of IL-6 due to decreased clearance. IL-6R-neutralizing antibodies interfere with binding of IL-6R to IL-6 (green; Table 1) and also block both classical and trans-signaling of IL- 6 but only lead to moderate elevation of IL- 6 levels due to impaired internalization and subsequent degradation of IL-6. The sgp130Fc protein (red/brown; Table 1) specifically blocks IL-6 trans-signaling without affecting classical signaling via the membrane-bound IL-6R, since IL-6 alone has no measurable affinity to sgp $130 \mathrm{Fc}$.

and pain. Our view is that IL- 6 trans-signaling acts as a danger signal, which enhances IL- 6 responsiveness and drives inflammatory events. For example, sIL-6R is shed very rapidly from infiltrating neutrophils in response to chemotactic factors (chemokines, complement components, lipid mediators), CRP, and apoptosis activation, while localized increases in sIL-6R correlate with leukocyte infiltration and tissue damage (67-75). In contrast, classical IL-6R signaling coordinates the more homeostatic properties of IL-6, which possibly reflects its early description as a cytokine with hormone-like characteristics $(5,14)$.

A thorough understanding of the in vivo relevance of IL- 6 transsignaling came from the observation that a soluble form of gp130 (sgp130) selectively inhibits IL-6 trans-signaling without affecting the classical pathway $(76,77)$. Relatively high circulating concentrations of sgp130 $(100-300 \mathrm{ng} / \mathrm{ml})$ are detected in human sera, and production of this natural antagonist is governed by differential gp130 mRNA splicing, which generates 4 distinct sgp130 isoforms $(59,63)$. sgp130 has no measurable affinity for IL-6 or IL-6R alone (77). Instead, sgp130 only binds the IL-6/sIL-6R complex and therefore only blocks IL- 6 trans-signaling $(76,77)$. Using sgp130 as a molecular tool, various in vivo studies have now documented roles of IL-6 trans-signaling in experimental models of arthritis, colitis, infection, allergy, and inflammation-induced cancer $(71,75,76$, 78-84). For details regarding these activities, see refs. 5, 31, 52, 63.

Interestingly, the IL- 6 homolog viral IL-6, encoded by human herpes virus 8 , directly binds and activates gp130 in a manner similar to that of the IL-6/sIL-6R complex $(85,86)$. This finding not only underscores the importance of IL- 6 mimicry in vivo but also emphasizes the central physiological importance of IL-6 trans-signaling. It is not, however, clear why IL- 6 uses two distinct modes of receptor signaling. As a soluble cytokine receptor, sIL-6R has been proven to prolong the signaling activity and circulating half-life of IL-6 (62). Indeed, for cells lacking IL-6R (but expressing gp130), IL- 6 trans-signaling remains the only mechanism by which these cells respond to IL-6. sIL-6R levels are therefore rate limiting and prevent an inadvertent IL- 6 activation of endothelial cells and fibroblasts. In cell types expressing membrane-bound IL-6R, the situation may be slightly more complex. Here the surface expression of gp130 is generally greater than that of IL-6R, and trans-signaling can amplify the IL- 6 signal through increased gp130 engagement $(62,64)$. IL-6R is expressed at a higher level in CD4 ${ }^{+} \mathrm{T}$ cells than $\mathrm{CD}^{+} \mathrm{T}$ cells and is closely linked with the expression of CCR7 and $\mathrm{CD} 62 \mathrm{~L}$ in $\mathrm{CD}^{+} \mathrm{T}$ cells (i.e., naive or central memory $\mathrm{T}$ cell populations) (33). In contrast, effector cells from sites of inflammation lack IL-6R, and pan-TCR activation increases IL-6R shedding $(33,87,88)$. Consequently, activated $\mathrm{CD} 44^{\mathrm{hi}} \mathrm{CD} 62 \mathrm{~L}^{\mathrm{lo}} \mathrm{CD} 4^{+}$ T cells lose their capacity to respond to classical IL- 6 activation (33), yet these cells still respond to IL-6 trans-signaling, which promotes the expression of antiapoptotic regulators, such as Bcl-2 and $\mathrm{Bcl}-\mathrm{xl}(33,87)$. However, IL- 6 trans-signaling is unable to drive Th17 production in preactivated $\mathrm{CD}^{+} \mathrm{T}$ cells (33). Instead, IL-6 trans-signaling helps to maintain the effector characteristics of already precommitted Th17 cells (33). Although the mechanism for this response remains unclear, the level of IL-6R expression on T cells has been associated with changes in IL-6-mediated STAT1 but not STAT3 signaling (89). These data suggest an activationinduced alteration in IL- 6 responsiveness. While these changes have been linked to the regulation of T cell apoptosis, it is conceivable that such alterations in STAT signaling may also influence the control of T cell commitment. Similar data were also observed in FoxP3 regulatory $\mathrm{T}$ cells, suggesting that classical IL-6R signaling and IL-6 trans-signaling can orchestrate both similar and distinct T cell responses $(33,90,91)$.

\section{How does gp130 signaling drive disease?}

The central signaling molecule activated by gp130 is the latent transcription factor STAT3, which is phosphorylated by JAK proteins constitutively bound to the cytoplasmic portion of gp130. STAT3-deficient animals are not viable after embryonic day 7.5, and conditional deletion of the STAT3 gene in bone marrow cells during hematopoiesis causes Crohn disease-like pathology, overt

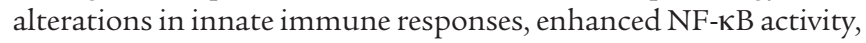
and increased lethality at 4-6 weeks $(92,93)$. These studies demonstrate that STAT3 is an integral component of development, inflammation, and cancer (94).

The clinical efficacy of tocilizumab suggests that IL-6/STAT3 signaling actively contributes to the pathology of autoimmune disorders, including rheumatoid arthritis (2). Activated STAT3 is detected at high levels in diseased tissues such as synovial biopsies from patients with rheumatoid arthritis $(3,95)$. In models of arthritis, levels of activated STAT3 are rapidly increased after disease induction and localized within the synovial lining and $\mathrm{CD}^{+}$ T cell clusters $(54,79)$. Indeed, STAT3 stimulation via IL-6/sIL-6R promotes synovial hyperplasia, joint erosion, chemokine-directed leukocyte recruitment, and the maintenance of effector cells within the inflamed joint $(36,54,78,79,96,97)$. To mechanistically link the control of disease processes with IL-6/STAT3 signaling, an increasing number of studies have used a gp130 knockin mouse 
model in which an amino acid substitution prevents feedback inhibition of the receptor, resulting in exaggerated STAT3 signaling $(79,98,99)$. In these systems, monoallelic deletion of Stat 3 led to a reduction in inflammation and overall pathogenesis (79). However, IL-6/STAT3 involvement has received the most attention in the field of tumor biology.

\section{Perspectives on cancer}

STAT3 activity often correlates with tumorigenesis and is associated with tumor growth, survival, angiogenesis, and metastatic processes, including epithelial-mesenchymal transition, degradation of extracellular matrix, and cell migration $(100,101)$. Each of these processes can be linked experimentally to gp130 signaling (4). For instance, in murine models of inflammation-induced colorectal cancer, STAT3-dependent tumorigenesis has been associated with both the local secretion of IL-6 $(102,103)$ and regulation of IL-6 trans-signaling $(80,83)$ within the tumor microenvironment. These studies have identified a link between IL- 6 and tumor-associated inflammation. Indeed, STAT3 activation in an oncogenic K-Rasdriven pancreatic tumor model does not develop spontaneously but is instead regulated by IL-6 and sIL-6R from myeloid tumor infiltrating cells (84). Similarly, it was recently shown in a newly developed model of ulcerative colitis-associated colon cancer that IL- 6 produced by M2-type macrophages via IL- 6 trans-signaling is involved in tumorigenesis (104). Interestingly, IL-6 was responsible for the higher prevalence of liver cancer in male littermates in this model (105). Although many studies have identified IL-6 as a major tumor-associated cytokine, IL-11 might also contribute to inflammation-induced cancer, as suggested from a study on gp130 signaling in gastric cancer (106). These underlying themes are also evident in human cancers in which IL-6/STAT3 activity is associated with tumor progression and poor prognosis. For example, in hepatocellular adenoma, somatic mutations coding for constitutively activated gp130 have been detected (107), while elevated IL-6 levels in patients with breast, lung, and hematopoietic tumors correlate with poor clinical outcome $(108,109)$. Biomarkers of STAT3 activity also represent reliable diagnostic/prognostic factors for patients with colorectal neoplasia and non-small cell lung carcinoma (100, 110-112). Furthermore, ADAM17, which mediates the ectodomain shedding (and activation) of some EGFR ligands, Notch and IL-6R, is also upregulated in various cancers (113) and is considered a novel anticancer drug target (114).

\section{How can gp130 be blocked?}

In the early 1990s, IL-6 was recognized as a major growth factor in multiple myeloma, and since that time, elevations in circulating IL-6 and sIL-6R levels have been used as prognostic indicators (reviewed in ref. 59). These studies led to clinical trials with neutralizing anti-IL- 6 antibodies, which showed good antitumor efficacy and a normalization of acute phase activity (115). However, antibody treatment led to massive systemic elevations (approaching mg quantities) in IL-6. Subsequent pharmacokinetic experiments revealed that antibody-associated IL- 6 was not cleared from the circulation, leading to a reservoir of free IL- 6 as concentrations reached the $\mathrm{K}_{\mathrm{D}}$ of the antibody (116). To overcome such difficulties, targeting strategies were redirected toward blockade of IL-6R. This led to development of tocilizumab, which prevents binding of IL-6 to IL-6R (117). Patients treated with tocilizumab show only a mild accumulation of IL-6, due to blockade of IL-6Rdependent internalization, which regulates IL-6 clearance (118).
The humanized antibody tocilizumab is now marketed as Actemra (RoActemra in the EU), which is approved for the treatment of rheumatoid arthritis in Europe and the US (Table 1). Other IL-6directed neutralizing antibodies are in clinical development as antiinflammatory and anticancer therapeutics (52), and these include human-mouse chimeric and fully humanized antibodies to human IL-6 (Table 1). However, each of these agents globally inhibits IL-6 activities, without differentiating between classical and trans-signaling (Figure 2).

The ubiquitous cellular expression of gp130 suggests that IL-6 trans-signaling has the potential to stimulate all cell types within the body. As noted above, this may in part be regulated by sgp130 variants, which circulate at high levels in human sera and selectively antagonize IL-6 trans-signaling (32). This finding has opened up the possibility of using sgp130 as a therapeutic modality for the treatment of inflammation. sgp130 linked to the Fc portion of IgG (sgp130Fc; ref. 77) is currently in preclinical development and shows efficacy in animal models of inflammatory arthritis, peritonitis, inflammatory bowel disease, and colon cancer $(8,52,71,76,78-80)$. These results suggest that during chronic disease progression, IL- 6 trans-signaling primarily drives the proinflammatory activities of IL-6. However, it is important to note that IL- 6 also governs certain antiinflammatory responses, including the resolution of innate immune responses, and the control of cytokine regulators, such as the soluble TNF receptor p55 and the IL-1 receptor antagonist $(71,119)$. The regulation of these activities may have important implications in the control of antimicrobial host defense and inflammation-associated tumor immunity $(31,71,102)$. The challenge will be to determine whether sgp130Fc offers a true clinical advantage over more standard mAb therapies against IL- 6 or IL-6R.

\section{Hurdles of gp130 blockade}

Due to the clinical successes of anti-TNF- $\alpha$ agents in treating inflammation, it is often difficult to promote the advantages of newer, alternative anti-cytokine-based biologic treatments. For example, the mechanism of action of tocilizumab is regularly compared with the mechanism of action of adalimumab or etanercept. However, not all patients respond to anti-TNF- $\alpha$ therapy, and approximately $45 \%$ of patients with inflammatory arthritis show poor response to treatment. Interestingly, a phase III trial in patients with rheumatoid arthritis failing anti-TNF- $\alpha$ therapy showed that certain individuals display increased responsiveness to tocilizumab $(120,121)$. Consequently, TNF- $\alpha$ and IL- 6 may have unique roles in inflammatory arthritis. This does not fit with the traditional view that TNF- $\alpha$ is upstream of IL- 6 in an inflammatory cytokine cascade. Such models probably do not reflect the complexity of the in vivo situation and were outlined prior to our increasing understanding of IL- 6 trans-signaling. In support of this, it has been demonstrated that TNF- $\alpha$ does not induce IL-6R shedding (122). As alternative anti-IL-6-based modalities (Table 1) filter into the clinic, their efficacies will need to be distinguished from those of the anti-TNF- $\alpha$ blockers and tocilizumab. While these are challenging clinical considerations, a recent murine study of IL-6 involvement in sepsis provides an example of the type of thinking required. Using a cecal ligation and puncture sepsis model, the authors showed that global blockade of IL- 6 by neutralizing antibodies was not beneficial, whereas selective inhibition of IL-6 trans-signaling resulted in increased animal survival (123). A possible explanation for these findings lies in the recent apprecia- 


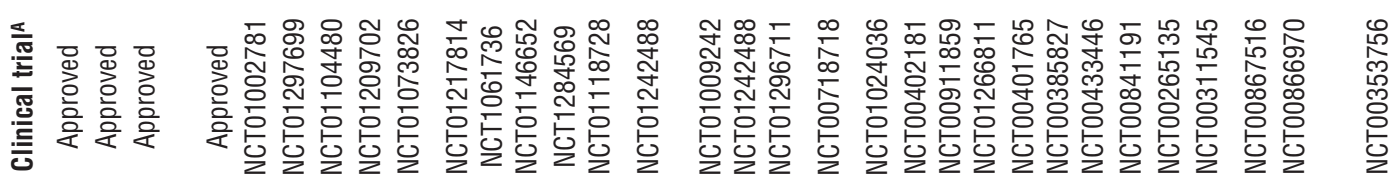

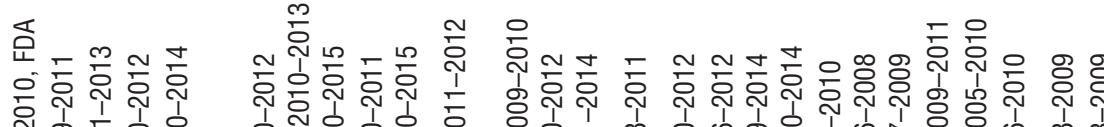

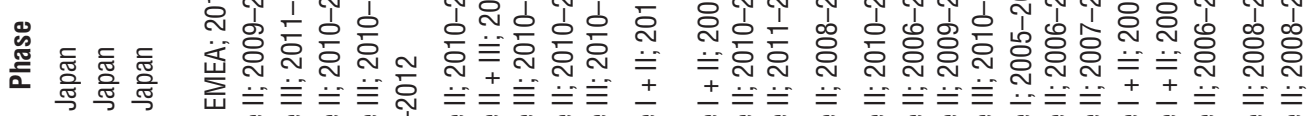

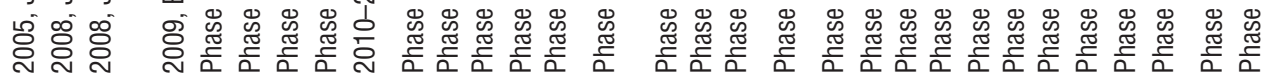
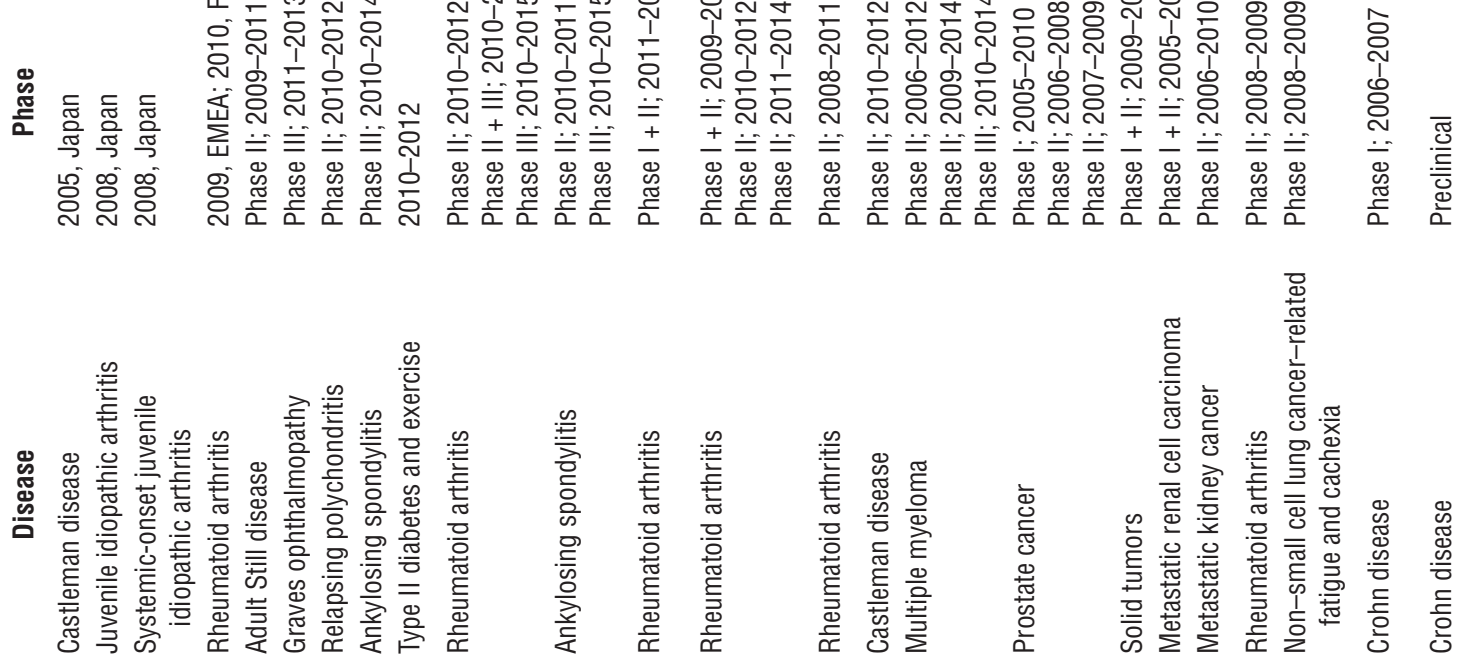

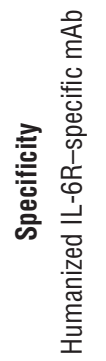
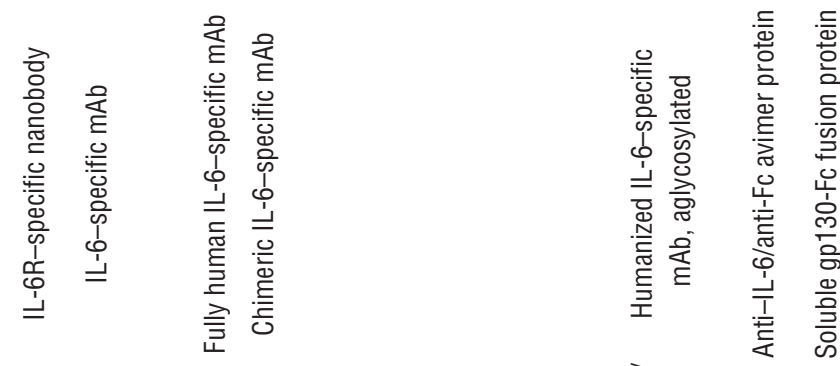

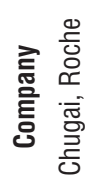
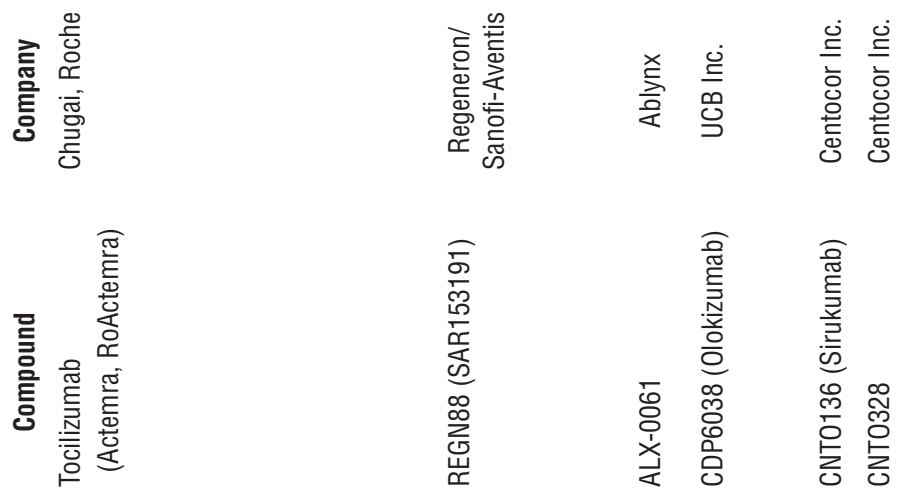

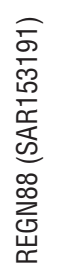

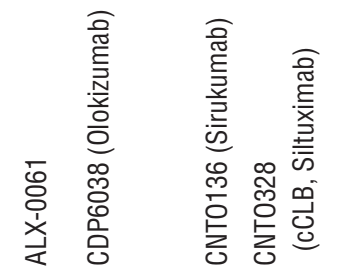

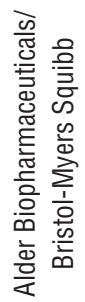
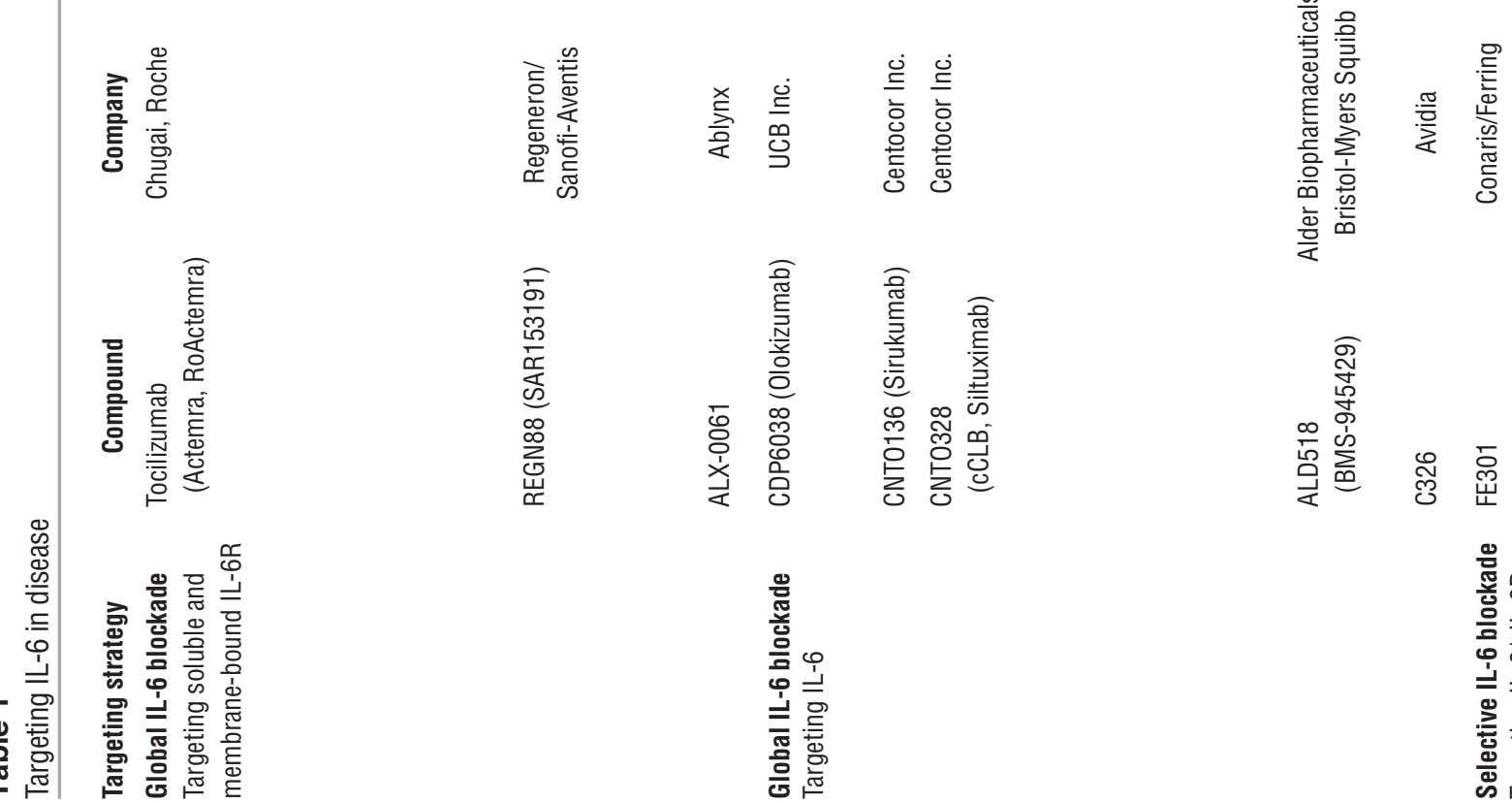

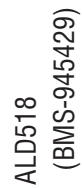

্ָণ 
tion that intestinal epithelial cells express membrane-bound IL-6R (80) and that IL-6 signaling and STAT3 activation in these cells drives epithelial regeneration (102). When IL-6 trans-signaling is blocked by sgp $130 \mathrm{Fc}$, free IL- 6 is not neutralized and directly stimulates STAT3 in intestinal epithelial cells via classical IL-6R signaling, leading to regeneration of the epithelium $(102,103)$. In light of this, it is interesting to note that tocilizumab is not recommended for patients with a preexisting history of diverticulitis. Although these are rare occurrences and tocilizumab displays a robust safety profile $(1,66)$, it will be important to better understand how IL-6 influences homeostatic processes, such as neutropenia, changes in cholesterol, and weight gain, which have been linked with tocilizumab intervention (2).

\section{Future perspectives}

Remission of disease and prevention of irreversible tissue damage remains the ultimate objective for treatment of inflammatory conditions like rheumatoid arthritis. To achieve this goal it is evident that appropriate early intervention is the most effective therapeutic strategy (124). However, clinical criteria alone are often inadequate to identify patients with rapidly progressing disease or predict the likely course of an inflammatory condition (124). As newer alternative biologics and small molecule inhibitors become clinically available, selecting the most appropriate treatment for an individual patient becomes more complex. So how do we improve clinical decisions on the best choice of drug for an individual patient? In the context of IL- 6 biology, we need to understand how gp130 signaling in acute resolving inflammation becomes distorted to instead drive chronic disease. The regulation of STAT3 by IL- 6 has received considerable attention in the study of both cancer biology and (auto)immunity, and pathway signatures that reflect altered STAT3 activity have prognostic value in certain cancers (4, 110-112). Furthermore, pharmacogenomic approaches have identified genetic links between STAT3 and chronic disease. For example, meta-analysis of a genome-wide association study of a European patient cohort identified seven new rheumatoid arthritis risk loci. These included gene products associated with STAT3 signaling/activity (IL6ST [GP130], SPRED2, CCR6), while a further suggestive risk allele was noted in the IL6R gene (125). Future studies will, however, need to take a more integrated view to validate the functional impact of these risk loci. Ideally, this should include their impact on chronic disease progression and secondary outcomes associated with biologic interventions, such as plasma lipid profiles, infection incidence, mood, fatigue, and malignancy (2).

In summary, interventions directed against IL-6/gp130 signaling represent excellent targets for therapy. At present, the application of these drugs has been restricted to certain inflammatory conditions; however, as evidenced by the number of anti-IL- 6 based modalities currently under clinical development (Table 1), this is likely to broaden over coming years. The emerging challenge is to know how best to target this inflammatory pathway and how to identify patients that may benefit most from IL-6-blocking therapies.

\section{Acknowledgments}

S. Rose-John and J. Scheller are funded by grants from the Deutsche Forschungsgemeinschaft, Bonn, Germany (SFB654, projects C5, C8; SFB841, project C1; SFB877, projects A1, A2) and by the Cluster of Excellence "Inflammation at Interfaces." S.A. Jones is supported by grants from Arthritis Research UK $(18286,19381)$ and The Wellcome Trust and through an Arthritis Research UK Centre grant (18461) in Biomechanics and Bioengineering.

Address correspondence to: Stefan Rose-John, Institute of Biochemistry, Christian-Albrechts-University, Olshausenstrasse 40, D-24098 Kiel, Germany. Phone: 49.431.880.3336; Fax: 49.431.880.5007; E-mail: rosejohn@biochem.uni-kiel.de.
1. Feldmann M. Development of anti-TNF therapy for rheumatoid arthritis. Nat Rev Immunol. 2002; 2(5):364-371.

2. McInnes IB, Schett G. Cytokines in the pathogenesis of rheumatoid arthritis. Nat Rev Immunol. 2007;7(6):429-442.

3. Ivashkiv LB, Hu X. The JAK/STAT pathway in rheumatoid arthritis: pathogenic or protective? Arthritis Rheum. 2003;48(8):2092-2096.

4. Grivennikov SI, Karin M. Dangerous laison: STAT3 and NF-kappaB collaboration and crosstalk in cancer. Cytokine Growth Factor Rev. 2010;21(1):11-19.

5. Kishimoto T. IL-6: from its discovery to clinical applications. Int Immunol. 2010;22(5):347-352.

6. Choy E. RADIATE: more treatment options for patients with an inadequate response to tumor necrosis factor antagonists. Nat Clin Pract Rheumatol. 2009;5(2):66-67.

7. Kremer JM, et al. The safety and efficacy of a JAK inhibitor in patients with active rheumatoid arthritis: Results of a double-blind, placebo-controlled phase IIa trial of three dosage levels of CP-690,550 versus placebo. Arthritis Rheum. 2009;60(7):1895-1905.

8. Melton L, Coombs A. Actemra poised to launch IL-6 inhibitors. Nat Biotechnol. 2008;26(9):957-959.

9. Yoshida K, et al. Targeted disruption of gp130, a common signal transducer for the interleukin 6 family of cytokines, leads to myocardial and hematological disorders. Proc Natl Acad Sci U S A. 1996; 93(1):407-411.

10. Baumann H, Schendel P. Interleukin-11 regulates the hepatic expression of the same plasma protein genes as interleukin-6. J Biol Chem. 1991; 266(30):20424-20427.
11. DeChiara TM, et al. Mice lacking the CNTF receptor, unlike mice lacking CNTF, exhibit profound motor neuron deficits at birth. Cell. 1995;83(2):313-322.

12. Elson GC, et al. CLF associates with CLC to form a functional heteromeric ligand for the CNTF receptor complex. Nat Neurosci. 2000;3(9):867-872.

13. Escary JL, Perreau J, Dumenil D, Ezine S, Brulet P. Leukaemia inhibitory factor is necessary for maintenance of haematopoietic stem cells and thymocyte stimulation. Nature. 1993;363(6427):361-364.

14. Heinrich PC, Behrmann I, Haan S, Hermanns HM, Muller-Newen G, Schaper F. Principles of interleukin (IL)-6-type cytokine signalling and its regulation. Biochem J. 2003;374(pt 1):1-20.

15. Kopf $M$, et al. Impaired immune and acute-phase responses in interleukin-6-deficient mice. Nature. 1994;368(6469):339-342.

16. Pennica D, et al. Cardiotrophin-1. Biological activities and binding to the leukemia inhibitory factor receptor/gp130 signaling complex. J Biol Chem. 1995;270(18):10915-10922.

17. Pflanz S, et al. WSX-1 and glycoprotein 130 constitute a signal-transducing receptor for IL-27. J Immunol. 2004;172(4):2225-2231.

18. Robb L, Li R, Hartley L, Nandurkar HH, Koentgen $\mathrm{F}$, Begley CG. Infertility in female mice lacking the receptor for interleukin 11 is due to a defective uterine response to implantation. Nat Med. 1998; 4(3):303-308

19. Stewart CL. Leukaemia inhibitory factor and the regulation of pre-implantation development of the mammalian embryo. Mol Reprod Dev. 1994; 39(2):233-238.

20. Kallen K-J. The role of transsignalling via the ago- nistic soluble IL-6 receptor. Biochim Biophys Acta. 2002;1592(3):323-343.

21. Campbell IK, O’Donnell K, Lawlor KE, Wicks IP. Severe inflammatory arthritis and lymphadenopathy in the absence of TNF. J Clin Invest. 2001; 107(12):1519-1527.

22. Ji H, et al. Critical roles for interleukin 1 and tumor necrosis factor alpha in antibody-induced arthritis. J Exp Med. 2002;196(1):77-85.

23. Liu J, et al. TNF is a potent anti-inflammatory cytokine in autoimmune-mediated demyelination. Nat Med. 1998;4(1):78-83.

24. Ohshima $S$, et al. Interleukin-6 plays a key role in the development of antigen-induced arthritis. Proc Natl Acad Sci U S A. 1998;95(14):8222-8226.

25. Alonzi $\mathrm{T}$, et al. Interleukin 6 is required for the development of collagen-induced arthritis. J Exp Med. 1998;187(4):461-468.

26. Richards HB, Satoh M, Shaw M, Libert C, Poli V, Reeves WH. Interleukin 6 dependence of anti-DNA antibody production: evidence for two pathways of autoantibody formation in pristane-induced lupus. J Exp Med. 1998;188(5):985-990.

27. Eugster HP, Frei K, Kopf M, Lassmann H, Fontana A. IL-6-deficient mice resist myelin oligodendrocyte glycoprotein-induced autoimmune encephalomyelitis. Eur J Immunol. 1998;28(7):2178-2187.

28. Screpanti I, et al. Inactivation of the IL6-gene prevents development of multicentric Castleman's disease in C/EBP $\beta$-deficient mice. J Exp Med. 1996; 184(4):1561-1566.

29. Lattanzio G, et al. Defective development of pristaneoil-induced plasmacytomas in interleukin-6-deficient BALB/c mice. Am J Pathol. 1997;151(3):689-696. 
30. Rose-John S, Heinrich PC. Soluble receptors for cytokines and growth factors: generation and biological function. Biochem J. 1994;300(pt 2):281-290.

31. Jones SA. Directing transition from innate to acquired immunity: Defining a role for IL-6. J Immunol. 2005;175(6):3463-3468.

32. Rose-John S, Scheller J, Elson G, Jones S. Interleukin- 6 biology is coordinated by membrane-bound and soluble receptors: role in inflammation and cancer. J Leukoc Biol. 2006;80(2):227-236.

33. Jones GW, et al. Loss of CD4+ T cell IL-6R expression during inflammation underlines a role for IL-6 trans signaling in the local maintenance of Th17 cells. J Immunol. 2010;184(4):2130-2139.

34. McFarland-Mancini MM, et al. Differences in wound healing in mice with deficiency of IL- 6 versus IL-6 receptor. J Immunol. 2010;184(12):7219-7228.

35 . Wunderlich FT, et al. Interleukin-6 signaling in liver-parenchymal cells suppresses hepatic inflammation and improves systemic insulin action. Cell Metab. 2010;12(3):237-249.

36. Wong PK, Quinn JM, Sims NA, van Nieuwenhuijze A, Campbell IK, Wicks IP. Interleukin- 6 modulates production of $\mathrm{T}$ lymphocyte-derived cytokines in antigen-induced arthritis and drives inflammationinduced osteoclastogenesis. Arthritis Rheum. 2006; 54(1):158-168

37. Kalliolias GD, Zhao B, Triantafyllopoulou A Park-Min KH, Ivashkiv LB. Interleukin-27 inhibits human osteoclastogenesis by abrogating RANKLmediated induction of nuclear factor of activated $\mathrm{T}$ cells $\mathrm{c} 1$ and suppressing proximal RANK signaling. Arthritis Rheum. 2010;62(2):402-413.

38. Niedbala W, et al. Interleukin 27 attenuates collagen-induced arthritis. Ann Rheum Dis. 2008; 67(10):1474-1479.

39. Hermann JA, Hall MA, Maini RN, Feldmann M, Brennan FM. Important immunoregulatory role of interleukin-11 in the inflammatory process in rheumatoid arthritis. Arthritis Rheum. 1998;41(8):1388-1397.

40. Walmsley M, Butler DM, Marinova-Mutafchieva L, Feldmann M. An anti-inflammatory role for interleukin-11 in established murine collagen-induced arthritis. Immunology. 1998;95(1):31-37.

41. Moreland L, et al. Results of a phase-I/II randomized, masked, placebo-controlled trial of recombinant human interleukin-11 (rhIL-11) in the treatment of subjects with active rheumatoid arthritis. Arthritis Res. 2001;3(4):247-252.

42. Sims NA. gp130 signaling in bone cell biology: multiple roles revealed by analysis of genetically altered mice. Mol Cell Endocrinol. 2009;310(1-2):30-39.

43. Nakamura K, Nonaka H, Saito H, Tanaka M, Miyajima A. Hepatocyte proliferation and tissue remodeling is impaired after liver injury in oncostatin $\mathrm{M}$ receptor knockout mice. Hepatology. 2004;39(3):635-644.

44. Hams E, et al. Oncostatin $\mathrm{M}$ receptor-beta signaling limits monocytic cell recruitment in acute inflammation. J Immunol. 2008;181(3):2174-2180.

45. Esashi E, Ito H, Minehata K, Saito S, Morikawa Y, Miyajima A. Oncostatin M deficiency leads to thymic hypoplasia, accumulation of apoptotic thymocytes and glomerulonephritis. Eur Immunol. 2009; 39(6):1664-1670

46. Matthews VB, et al. Interleukin-6-deficient mice develop hepatic inflammation and systemic insulin resistance. Diabetologia. 2010;53(11):2431-2441.

47. Chesnokova V, Melmed S. Neuro-immuno-endocrine modulation of the hypothalamic-pituitaryadrenal (HPA) axis by gp130 signaling molecules. Endocrinology. 2002;143(5):1571-1574.

48. Waage A, Brandtzaeg P, Halstensen A, Kierulf P, Espevik T. The complex pattern of cytokines in serum from patients with meningococcal septic shock. J Exp Med. 1989;169(1):333-338.

49. Veldhoen M, Hocking RJ, Atkins CJ, Locksley RM, Stockinger B. TGFbeta in the context of an inflammatory cytokine milieu supports de novo differen- tiation of IL-17-producing T cells. Immunity. 2006; 24(2):179-189.

50. McKenzie BS, Kastelein RA, Cua DJ. Understanding the IL-23-IL-17 immune pathway. Trends Immunol. 2006;27(1):17-23.

51. Roll P, et al. In vivo effects of the anti-interleukin- 6 receptor inhibitor tocilizumab on the B cell compartment. Arthritis Rheum. 2011;63(5):1255-1264.

52. Kopf M, Bachmann MF, Marsland BJ. Averting inflammation by targeting the cytokine environment. Nat Rev Drug Discov. 2010;9(9):703-718.

53. Modur V, et al. Oncostatin $\mathrm{M}$ is a proinflammatory mediator. In vivo effects correlate with endothelial cell expression of inflammatory cytokines and adhesion molecules. JClin Invest. 1997;100(1):158-168.

54. Nowell MA, et al. Regulation of pre-B cell colonyenhancing factor by STAT-3-dependent interleukin- 6 trans-signaling: implications in the pathogenesis of rheumatoid arthritis. Arthritis Rheum. 2006;54(7):2084-2095.

55. Hunter CA. New IL-12-family members: IL-23 and IL-27, cytokines with divergent functions. Nat Rev Immunol. 2005;5(7):521-531.

56 . Stumhofer JS, et al. Interleukin 27 negatively regulates the development of interleukin 17-producing T helper cells during chronic inflammation of the central nervous system. Nat Immunol. 2006;7(9):937-945.

57. Stumhofer JS, et al. Interleukins 27 and 6 induce STAT3-mediated $\mathrm{T}$ cell production of interleukin 10. Nat Immunol. 2007;8(12):1363-1371

58. Stumhofer JS, et al. A role for IL-27p28 as an antagonist of gp130-mediated signaling. Nat Immunol. 2010;11(12):1119-1126

59. Jones SA, Horiuchi S, Topley N, Yamamoto N, Fuller GM. The soluble interleukin 6 receptor: mechanisms of production and implications in disease. FASEB J. 2001;15(1):43-58.

60. Honda M, et al. Human soluble IL- 6 receptor: its detection and enhanced release by HIV infection. Immunol. 1992;148(7):2175-2180.

61. Novick D, Engelmann H, Wallach D, Rubinstein M. Soluble cytokine receptors are present in normal human urine. J Exp Med. 1989;170(4):1409-1414.

62. Peters $M$, et al. The function of the soluble interleukin 6 (IL-6) receptor in vivo: sensitization of human soluble IL-6 receptor transgenic mice towards IL-6 and prolongation of the plasma half-life of IL-6. J Exp Med. 1996;183(4):1399-1406.

63. Jones S, Richards PJ, Scheller J, Rose-John S. IL-6 transsignaling: the in vivo consequences. J Interferon Cytokine Res. 2005;25(5):241-253.

64. Peters $M$, et al. Soluble IL-6 receptor leads to a paracrine modulation of the hepatic acute phase response in double transgenic mice. Jimmunol. 1997; 159(3):1474-1481.

65. Gearing DP, Cosman D. Homology of the p40 subunit of natural killer cell stimulatory factor (NKSF) with the extracellular domain of the interleukin-6 receptor. Cell. 1991;66(1):9-10.

66. Nishimoto N, et al. Humanized anti-interleukin-6 receptor antibody treatment of multicentric Castleman disease. Blood. 2005;106(8):2627-2632.

67. Kotake S, et al. Interleukin-6 and soluble interleukin-6 receptors in the synovial fluids from rheumatoid arthritis patients are responsible for osteoclast-like cell formation. J Bone Miner Res. 1996;11(1):88-95.

68. Desgeorges A, et al. Concentrations and origins of soluble interleukin 6 receptor-alpha in serum and synovial fluid. J Rheumatol. 1997;24(8):1510-1516.

69. Modur V, Li Y,Zimmerman GA, Prescott SM, McIntyre TM. Retrograde inflammatory signaling from neutrophils to endothelial cells by soluble interleukin- 6 receptor alpha. J Clin Invest. 1997;100(11):2752-2756.

70. Jones SA, Novick D, Horiuchi S, Yamamoto N, Szalai AJ, Fuller GM. C-reactive protein: a physiological activator of interleukin 6 receptor shedding. J Exp Med. 1999;189(3):599-604.

71. Hurst SM, et al. IL-6 and its soluble receptor orches- trate a temporal switch in the pattern of leukocyte recruitment. Immunity. 2001;14(6):705-714.

72. Marin V, et al. The IL-6-soluble IL-6Ralpha autocrine loop of endothelial activation as an intermediate between acute and chronic inflammation: an experimental model involving thrombin. J Immunol. 2001;167(6):3435-3442.

73. Marin V, Montero-Julian FA, Gres S, Bongrand P, Farnarier C, Kaplanski G. Chemoktactic agents induce IL-6Ra shedding from polymorphonuclear cells: involvement of a metalloprotease of the TNFalpha-converting enzyme (TACE) type. Eur Immunol. 2002;32(10):2965-2972.

74. McLoughlin RM, et al. Differential regulation of neutrophil-activating chemokines by IL- 6 and its soluble receptor isoforms. J Immunol. 2004; 172(9):5676-5683.

75. Chalaris A, et al. Apoptosis is a natural stimulus of IL6R shedding and contributes to the pro-inflammatory trans-signaling function of neutrophils. Blood. 2007;110(6):1748-1755.

76. Atreya R, et al. Blockade of IL- 6 transsignaling abrogates established experimental colitis in mice by suppression of the antiapoptotic resistance of lamina propria T cells. Nat Med. 2000;6(5):583-588

77. Jostock T, et al. Soluble gp130 is the natural inhibitor of soluble IL-6R transsignaling responses. Eur J Biochem. 2001;268(1):160-167.

78. Nowell MA, et al. Soluble IL-6 receptor governs IL- 6 activity in experimental arthritis: blockade of arthritis severity by soluble glycoprotein 130 . J Immunol. 2003;171(6):3202-3209.

79. Nowell MA, et al. Therapeutic targeting of IL-6 trans-signaling counteracts STAT3 control of the inflammatory infiltrate in experimental arthritis. J Immunol. 2009;182(1):613-622.

80. Becker C, et al. TGF-beta suppresses tumor progression in colon cancer by inhibition of IL- 6 transsignaling. Immunity. 2004;21(4):491-501.

81. Richards PJ, et al. Functional characterization of a soluble gp130 isoform and its therapeutic capacity in an experimental model of inflammatory arthritis. Arthritis Rheum. 2006;54(5):1662-1672.

82. Rose-John S, Waetzig GH, Scheller J, Grotzinger J, Seegert D. The IL-6/sIL-6R complex as a novel target for therapeutic approaches. Expert Opin Ther Targets. 2007;11(5):613-624.

83. Matsumoto S, et al. Essential roles of IL-6 transsignaling in colonic epithelial cells, induced by the IL-6/soluble-IL-6 receptor derived from lamina propria macrophages, on the development of colitisassociated premalignant cancer in a murine model. J Immunol. 2010;184(3):1543-1551.

84. Lesina M, et al. Stat3/Socs3 activation by IL-6 transsignaling promotes progression of pancreatic intraepithelial neoplasia and development of pancreatic cancer. Cancer Cell. 2011;19(4):456-469.

85. Müllberg J, et al. IL-6-receptor independent stimulation of human gp130 by viral IL-6. J Immunol. 2000;164(9):4672-4677.

86. Fielding CA, et al. Viral IL-6 blocks neutrophil infiltration during acute inflammation.J Immunol. 2005; 175(6):4024-4029.

87. Curnow SJ, et al. Inhibition of $\mathrm{T}$ cell apoptosis in the aqueous humor of patients with uveitis by IL-6/ soluble IL-6 receptor trans-signaling. J Immunol. 2004;173(8):5290-5297.

88. Briso EM, Dienz O, Rincon M. Cutting edge: soluble IL-6R is produced by IL-6R ectodomain shedding in activated CD4 T cells. J Immunol. 2008; 180(11):7102-7106.

89. Teague TK, et al. Activation-induced inhibition of interleukin 6-mediated T cell survival and signal transducer and activator of transcription 1 signaling. J Exp Med. 2000;191(6):915-926.

90. Doganci A, et al. The IL-6R alpha chain controls lung $\mathrm{CD} 4{ }^{+} \mathrm{CD} 4{ }^{+} \mathrm{CD} 25^{+} \mathrm{T}$ regulatory cell development and function during allergic airway inflam- 
mation in vivo. J Clin Invest. 2005;115(2):313-325

91. Dominitzki S, et al. Cutting edge: trans-signaling via the soluble IL-6R abrogates the induction of FoxP3 in naive CD4+CD25 T cells. J Immunol. 2007;179(4):2041-2045.

92. Akira S. Roles of STAT3 defined by tissue-specific gene targeting. Oncogene. 2000;19(21):2607-2611.

93. Welte $T$, et al. STAT3 deletion during hematopoiesis causes Crohn's disease-like pathogenesis and lethality: A critical role of STAT3 in innate immunity. Proc Natl Acad Sci U S A. 2003;100(4):1879-1884.

94. Bromberg J. Stat proteins and oncogenesis. J Clin Invest. 2002;109(9):1139-1142.

95. Walker JG, Smith MD. The Jak-STAT pathway in rheumatoid arthritis. J Rheumatol. 2005; 32(9):1650-1653

96. de Hooge AS, et al. Local activation of STAT-1 and STAT- 3 in the inflamed synovium during zymosaninduced arthritis: exacerbation of joint inflammation in STAT-1 gene-knockout mice. Arthritis Rheum. 2004;50(6):2014-2023.

97. Lissilaa R, et al. Although IL- 6 trans-signaling is sufficient to drive local immune responses, classical IL- 6 signaling is obligate for the induction of $\mathrm{T}$ cell-mediated autoimmunity. J Immunol. 2010; 185(9):5512-5521.

98. Jenkins BJ, et al. Hyperactivation of Stat 3 in gp 130 mutant mice promotes gastric hyperproliferation and desensitizes TGF-beta signaling. Nat Med.2005; 11(8):845-852.

99. Fielding CA, et al. IL- 6 regulates neutrophil trafficking during acute inflammation via STAT3. J Immunol. 2008;181(3):2189-2195.

100.Regis G, Pensa S, Boselli D, Novelli F, Poli V. Ups and downs: the STAT1:STAT3 seesaw of Interferon and gp130 receptor signalling. Semin Cell Dev Biol. 2008;19(4):351-359.

101.Yu H, Pardoll D, Jove R. STATs in cancer inflammation and immunity: a leading role for STAT3. Nat Rev Cancer. 2009;9(11):798-809.

102. Grivennikov S, et al. IL-6 and STAT3 signaling is required for survival of intestinal epithelial cells and colitis associated cancer. Cancer Cell. 2009; 16(2):103-113

103. Bollrath J, et al. gp130-mediated Stat 3 activation in enterocytes regulates cell survival and cell-cycle progression during colitis-associated tumorigenesis. Cancer Cell. 2009;15(2):91-102.

104.Schiechl G, et al. Tumor development in murine ulcerative colitis depends on MyD88 signaling of colonic $\mathrm{F} 4 / 80^{+} \mathrm{CD} 11 \mathrm{~b}^{\text {high }} \mathrm{Gr} 1^{\text {low }}$ macrophages. J Clin Invest. 2011;121(5):1692-1708.

105.Naugler WE, et al. Gender disparity in liver cancer due to sex differences in MyD88-dependent IL-6 production. Science. 2007;317(5834):121-124.

106. Ernst M, et al. STAT3 and STAT1 mediate IL-11 dependent and inflammation-associated gastric tumorigenesis in gp130 receptor mutant mice. JClin Invest. 2008;118(5):1727-1738.

107. Rebouissou S, et al. Frequent in-frame somatic deletions activate gp130 in inflammatory hepatocellular tumours. Nature. 2009;457(7226):200-204.

108. Bachelot T, Ray-Coquard I, Menetrier-Caux C, Rastkha M, Duc A, Blay JY. Prognostic value of serum levels of interleukin 6 and of serum and plasma levels of vascular endothelial growth factor in hormone-refractory metastatic breast cancer patients. BrJ Cancer. 2003;88(11):1721-1726.

109. Hong DS, Angelo LS, Kurzrock R. Interleukin-6 and its receptor in cancer: implications for translational therapeutics. Cancer. 2007;110(9):1911-1928.

110.Jackson CB, Giraud AS. STAT3 as a prognostic marker in human gastric cancer. $J$ Gastroenterol Hepatol. 2009;24(4):505-507.

111. Kim DY, et al. STAT3 expression in gastric cancer indicates a poor prognosis. J Gastroenterol Hepatol. 2009;24(4):646-651.

112. Qu P, et al. Stat3 downstream genes serve as biomarkers in human lung carcinomas and chronic obstructive pulmonary disease. Lung Cancer. 2009; 63(3):341-347.

113. Kenny PA, Bissell MJ. Targeting TACE-dependent EGFR ligand shedding in breast cancer. J Clin Invest. 2007; 117(2):337-345.

114. Scheller J, Chalaris A, Garbers C, Rose-John S. ADAM17: a molecular switch to control inflammation and tissue regeneration. Trends Immunol. 2011; 32(8):380-387.

115. Klein B, Lu ZY, Gaillard JP, Harousseau JL, Bataille R. Inhibiting IL-6 in human multiple myeloma. Curr Top Microbiol Immunol. 1992;182:237-244.

116. Lu ZY, Brochier J, Wijdenes J, Brailly H, Bataille R,
Klein B. High amounts of circulating interleukin (IL)-6 in the form of monomeric immune complexes during anti-IL-6 therapy. Towards a new methodology for measuring overall cytokine production in human in vivo. Eur J Immunol. 1992;22(11):2819-2824.

117. Sato K, et al. Reshaping a human antibody to inhibit the interleukin 6-dependent tumor cell growth. Cancer Res. 1993;53(4):851-856.

118. Nishimoto N, Terao K, Mima T, Nakahara H, Takagi N, Kakehi T. Mechanisms and pathologic significances in increase in serum interleukin-6 (IL-6) and soluble IL- 6 receptor after administration of an anti-IL-6 receptor antibody, tocilizumab, in patients with rheumatoid arthritis and Castleman disease. Blood. 2008;112(10):3959-3964.

119.Tilg H, Trehu E, Atkins MB, Dinarello CA, Mier JW. Interleukin-6 (IL-6) as an anti-inflammatory cytokine: induction of circulating IL-1 receptor antagonist and soluble tumor necrosis factor receptor p55. Blood. 1994;83(1):113-118.

120. Yokota S, et al. Efficacy and safety of tocilizumab in patients with systemic-onset juvenile idiopathic arthritis: a randomised, double-blind, placebocontrolled, withdrawal phase III trial. Lancet. 2008; 371(9617):998-1006.

121.Emery P, et al. IL-6 receptor inhibition with tocilizumab improves treatment outcomes in patients with rheumatoid arthritis refractory to anti-TNF biologics: results from a 24-week multicentre Randomised Placebo Controlled Trial. Ann Rheum Dis. 2008;67(11):1516-1523.

122. Jones SA, Horiuchi S, Novick D, Yamamoto N, Fuller GM. Shedding of the soluble IL- 6 receptor is triggered by $\mathrm{Ca} 2+$ mobilization, while basal release is predominantly the product of differential mRNA splicing in THP-1 cells. Eur J Immunol. 1998;28(11):3514-3522.

123. Barkhausen T, et al. Selective blockade of interleukin-6 trans-signaling improves survival in a murine polymicrobial sepsis model. Crit Care Med. 2011; 39(6):1407-1413.

124. Raza K, Filer A. Predicting the development of RA in patients with early undifferentiated arthritis. Best Pract Res Clin Rheumatol. 2009;23(1):25-36.

125.Stahl EA, et al. Genome-wide association study meta-analysis identifies seven new rheumatoid arthritis risk loci. Nat Genet. 2010;42(6):508-514. 